\title{
Optimal Self-assembly of Lipid Nanoparticles (LNP) in a Ring Micromixer
}

\section{Manon RIPOLL}

SANOFI, BioDPD department

\section{Elian Martin}

ESPCI Paris

\section{Mathilde Enot}

SANOFI, BioDPD department

\section{Oscar Robbe}

SANOFI, BioDPD department

\section{Chiara Rapisarda}

SANOFI, BioDPD department

Marie-Claire Nicolai

Sanofi-Pasteur

Aurelie Deliot

Sanofi-Pasteur

patrick tabeling ( $\square$ ptabeling30@gmail.com )

ESPCI Paris

Jean-René Authelin

SANOFI, BioDPD department

\section{Mostafa Nakach}

SANOFI, BioDPD department

\section{Pierre Wils}

SANOFI, BioDPD department

\section{Research Article}

Keywords:

Posted Date: February 24th, 2022

DOI: https://doi.org/10.21203/rs.3.rs-1343595/v1

License: (c) (1) This work is licensed under a Creative Commons Attribution 4.0 International License. Read Full License 



\title{
OPTIMAL SELF-ASSEMBLY OF LIPID NANOPARTICLES (LNP) IN A RING MICROMIXER
}

Manon Ripoll ${ }^{\mathrm{a}}$, Elian Martin ${ }^{\mathrm{b}}$, Mathilde Enot $^{\mathrm{a}}$, Oscar Robbe ${ }^{\mathrm{a}}$, Chiara Rapisarda ${ }^{\mathrm{a}}$, Marie-Claire Nicolai $^{\mathrm{c}}$, Aurélie Deliot ${ }^{\mathrm{c}}$, Patrick Tabeling ${ }^{\mathrm{b}^{*}}$, Jean-René Authelin ${ }^{\mathrm{a}}$, Mostafa Nakach ${ }^{\mathrm{a}}$, Pierre Wils ${ }^{\mathrm{a}}$

${ }^{\text {a }}$ SANOFI, BioDPD department, 13 Quai Jules Guesde, 94400 Vitry-sur-Seine.

${ }^{\mathrm{b}}$ Microfluidics, MEMS, Nanostructures Laboratory, CNRS Chimie Biologie Innovation (CBI) UMR 8231, Institut Pierre Gilles de Gennes (IPGG), ESPCI Paris, PSL Research University, 6 rue Jean Calvin 75005, Paris, France.

${ }^{\text {c} S A N O F I ~ P a s t e u r, ~ R E I ~ d e p a r t m e n t, ~} 1541$ Av. Marcel Mérieux, 69280 Marcy-l'Étoile

*Corresponding author : P.Tabeling, email : ptabeling30@gmail.com

\begin{abstract}
Lipid nanoparticles (LNPs) for RNA and DNA delivery have gained considerable attention to treat a broad range of diseases and to vectorize mRNA for COVID vaccines. LNPs are produced by mixing biomolecules and lipids, which self-assemble to form the desired structure. In this domain, microfluidics shows clear advantages: high mixing quality under low stress conditions added to fast preparation. Studies of LNPs produced in micromixers have revealed, in certain ranges of flow-rates, a degradation of performances in terms of size, size monodispersivity and encapsulation efficiency. In this study we focus on the ring micromixer, well adapted to high throughputs. We reveal three regimes - side-by-side, transitionnal and highly mixed - which control the mixing performance of the device. Further, on using cryo-TEM and biochemical analysis, we show that the mixing performances are strongly correlated to the characteristics of the LNPs we produce. We underline the important role of the flow-rate ratio and propose a physical criterion, based on the onset of temporal instabilities, for producing LNPs with optimal characteristics, in term of geometry, monodispersivity and encapsulation yield. These criteria are general.
\end{abstract}




\section{Introduction}

Much progress has been made, over the last decade, on microfluidic mixing, and, since the years where the first micromixers appeared in the literature, i.e around the turn of the century, about one hundred functional devices, based on a variety of concepts, have been invented. All have pros and cons, but, as a whole, users often find, in the microfluidic mixer catalog, geometries corresponding to the application they are interested $i^{1-3}$. In the recent years, the idea emerged using these devices for producing LNP (lipid nanoparticles) ${ }^{4,5}$. LNP have emerged as a gold standard for nucleic acid delivery ${ }^{6}$. They are complex nanoparticles, 50 to $100 \mathrm{~nm}$ in diameter, composed primarily of cationic ionizable lipids able to segregate from the other lipid components when charges are neutralized leading to the formation of amorphous oil droplet in the core of LNPs as described in a recent study ${ }^{7,8}$. The therapeutic molecule in the LNP depends on the application. It can be DNA, mRNA or siRNA. The functional entities entrapped with it, or adsorbed at the interface, include PEG moieties usually linked to a lipid chain, helper lipids, and cholesterol. Lipid nanoparticles offer many advantages over previous lipid-based nucleic acid delivery systems : high nucleic acid encapsulation efficiency and potent transfection, improved penetration into tissues along with low cytotoxicity and immunogenicity. These characteristics make lipid nanoparticles excellent candidates for nucleic acid delivery, as was demonstrated by the mRNA-based vaccines against COVID.

LNPs are formed through a self assembly process. Numerical simulations suggest that the self assembly process includes three steps: particle assembly into discoidal clusters, aggregation of clusters into larger membrane patches, and vesicle formation ${ }^{9}$. Self assembly would be too slow to achieve by diffusion (it would take days); thereby, hydrodynamic mixing is required. Using standard mixers ${ }^{5}$, in large containers, is an option. However, these mixers generate size polydispersivity along with low encapsulation efficiency. As a consequence, post processing steps, such as filtration, extrusion and centrifugation, are needed to improve the quality of the LNP produced in this manner,. In this context, the use of microfluidic mixers is pertinent. It has been recently showed that microfluidics allows to produce, in one step, with high yields, LNPs with acceptable quality, in terms of size monodispersivity and encapsulation yield. In Fujishima et al. ${ }^{10}$ and Shepherd et al. ${ }^{11}$, a staggered herringbone micromixer ${ }^{12}$ was used. One limitation of herringbone micromixers is the low throughput. This limit can be circumvented by parallelizing the system ${ }^{11}$. However, this option generates complexity, increases the cost and reduces the reliability. Inertial micromixers, which work at substantially larger flow-rates, and thus larger throughputs, offer a solution, but thus far, although 
some indications can be found in the literature, the question of the conditions under which they must be operated to obtain functional LNP has not been fully addressed yet.

In the present paper, we focus on a Dean-based micromixer ${ }^{13-17}$. This mixer belongs to the class of inertial micromixers. It is well adapted to high throughputs and thereby mass production. We identify, in good agreement with numerical calculations ${ }^{18}$, three domains of flow-rates. One of them, we call 'transition regime', will play an important role in our identification of the optimal flow conditions. To obtain LNPs with optimal characteristics, in term of encapsulation efficiency (EE), size and size monodispersivity, we show that we must operate, on a flow-rate scale, above the transition regime. Working below or inside the transition regime, still in terms of flow-rates, leads to degraded performances. In agreement with the literature, we thus outline the physical conditions under which LNP of acceptable quality can be obtained, using a physical criterion that has not been proposed thus far. We also discuss the important role of the aqueous phase (containing the nucleic acid) and the organic phase (containing the lipids) flow-rate ratio.

\section{Material and methods}

\subsection{LNP formulation}

In our LNP formulation, 1,2-dioleoyl-sn-glycero-3-phosphocholine (DOPC), 1,2-dimyristoyl-racglycero-3-methoxypolyethylene glycol-2000 (DMG-PEG2000) and cholesterol (plant derived) were purchased from Avanti Polar Lipids (Alabaster, AL, USA). Dlin-MC3-DNA named MC3 was obtained from SAI Life Science (Hyderabad, India). Citric acid and sodium citrate tribasic dehydrate were acquired from Sigma-Aldrich (France). Phosphate-buffered saline buffer 10X (PBS, pH 7,4) was obtained from Thermo Fisher Scientific (France). gWiz-GFP plasmid was purchased from Aldevron (North Dakota, USA).

To produce LNP, lipids were solubilized in ethanol at molar ratios of 50:10:38.5:1.5 respectively for MC3, DOPC, cholesterol and PEG-lipid. The lipid mixture was mixed with a $50 \mathrm{mM}$ citrate buffer at pH 4 containing the pDNA using the NxGen microfluidic cartridge (from Precision NanoSystems, Vancouver). The nitrogen to phosphate ratio (N/P) between the ionizable lipid and the pDNA was maintained at 6 . The impact of the flow rate (FR; ranging from 0.4 to $20 \mathrm{~mL} / \mathrm{min}$ ), the aqueous to organic ratio (FRR; from 1:1 to 10:1) and the final lipid and pDNA concentrations (from 1.44 to 15 $\mathrm{mg} / \mathrm{mL}$ and 93 to $965 \mu \mathrm{g} / \mathrm{mL}$ respectively for lipids and pDNA) were evaluated. For all formulations, initial and final waste volumes were set at 0.45 and $0.05 \mathrm{~mL}$ respectively. After the formulation is 
processed in the micromixer, ethanol is removed from the product and the citrate buffer exhanged by PBS using Amicon Ultra Centrifugal Filters (EMD Millipore, Billerica, MA). The formulations were finally passed through a $0.22-\mu \mathrm{m}$ filter and stored at $4{ }^{\circ} \mathrm{C}$ until use

\subsection{Measurements of nanoparticle size, polydispersity and zeta potential}

Particle size, polydispersity index (PDI) and zeta potential were measured by dynamic light scattering using the Malvern Zetasizer NanoZS (Worcestershire, UK). LNPs were diluted 100 times in PBS and added in $\mu$-cuve. The dispersant (PBS) refractive index (RI) and vicosity values were 1,335 and 1.02 $\mathrm{cP}$ whereas the material RI was 1.45 .

\subsection{Quantification of nucleic acid loading}

pDNA encapsulation efficiency was determined using the PicoGreen DNA assay (Life Technologies, Burlington, ON). Briefly, $100 \mu \mathrm{L}$ of the diluted fluorescent dye was added to $100 \mu \mathrm{L}$ of diluted LNP in the presence or absence of $1 \%(\mathrm{w} / \mathrm{v})$ Triton-X100 in TE buffer and incubated in absence of light for $5 \mathrm{~min}$. Nucleic acids were quantified by measuring fluorescence (ex/em $=480 \mathrm{~nm} / 520 \mathrm{~nm}$,) using a fluorimeter (Varioskan Lux Microplate reader, Thermo Fisher). A linear calibration curve up to $1000 \mathrm{ng} / \mathrm{mL}$ was perfomed using the standard DNA sample provided in the kit.

The encapsulation ratio was calculated by the following formula:

$$
\mathrm{EE}(\%)=\left(1-\frac{\text { pDNA concentration in absence of triton }}{\text { pDNA concentration in presence of triton }}\right) \times 100
$$

\subsection{Cryo TEM analysis}

The morphology of LNPs were observed by Cryo-electron microscopy. $4.1 \mu \mathrm{L}$ of concentrated LNPs were deposited on Qauntifoil R2/2 copper 300 mesh grids (quantifoil Instruments GmbH, Germany) after 90 seconds of glow discharge on an ELMO ionizer (Cordouan, France). Grids were blotted ad frozen using a Vitrobot MARK IV (ThermoFischer Scientific, USA) and transferred for observation onto a TEM tecnai-G20 (ThermoFisher Scientific, USA) operated at $200 \mathrm{KV}$ using a 910 cryo-holder (Gatan, Inc., USA). Images were recorded at $4 \mu \mathrm{m}$ defocus and at low dose mode (electron doses between 10 and 15 e-/Å2) using an ssCCD Ultrascan 4000 (Gatan, Inc., USA). Pixel size of recorded images was estimated to $0.221 \mathrm{~nm}$ after TEM calibration using a cross line grid (EMS, USA) with pitch spacing of $500 \mathrm{~nm}$ and 2000 lines/mm. 


\subsection{Microfluidic device, channel geometry and flow conditions}

The microfluidic device used to produce LNP is shown in Fig 1.
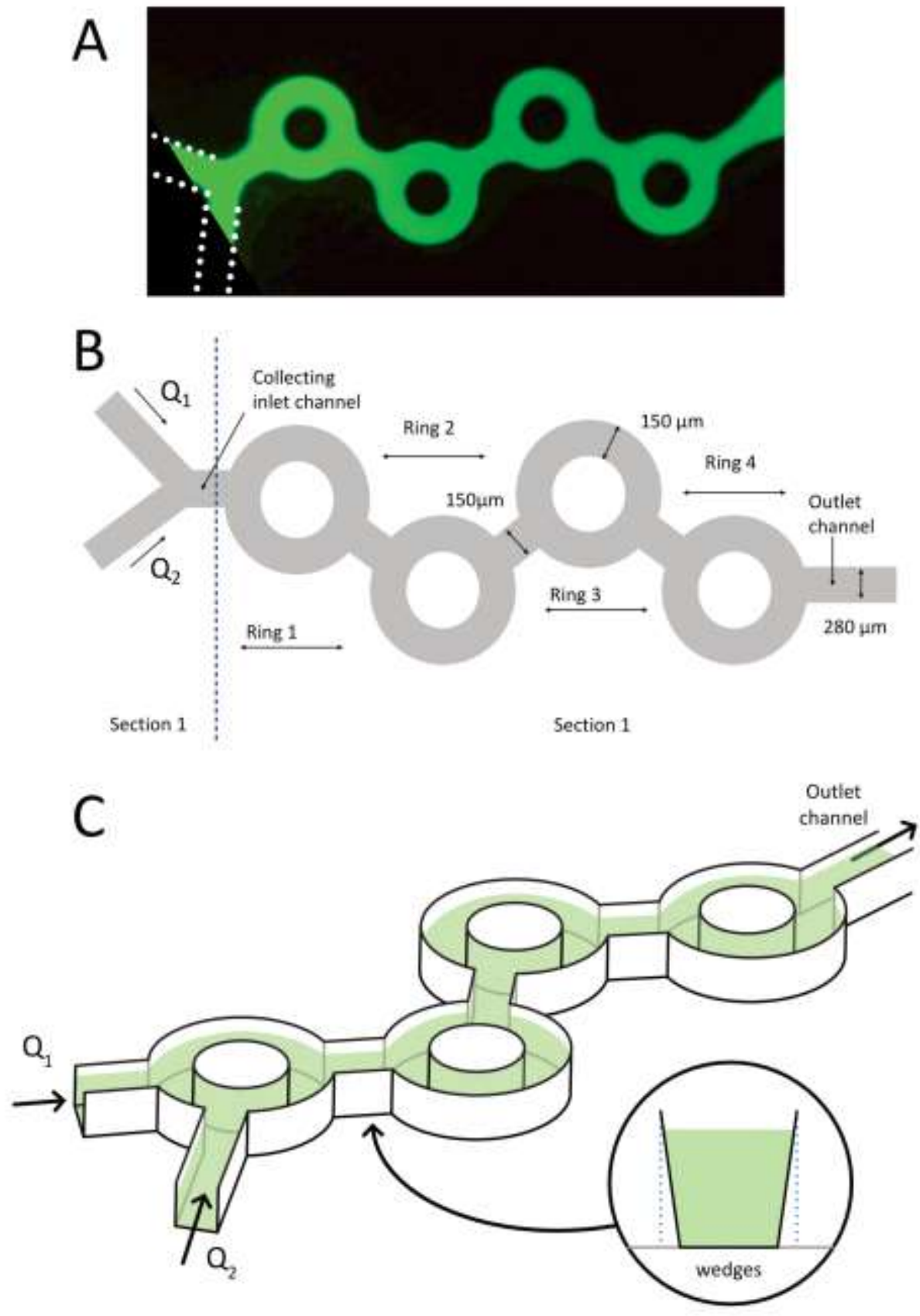

Fig 1: A - Image of the microdevice filled with a fluorescein solution. B - Sketch of the microdevice showing Section 1, the entry region, and Section 2, including the four rings and the junctions between them. In the fluid model experiments, $\mathrm{Q}_{1}$ is water and $\mathrm{Q}_{2}$ ethanol with $0.1 \%$ w/w fluorescein. In the LNP experiments, $\mathrm{Q}_{1}$ 
represents the aqueous phase containing the nucleic acid diluted in an acidic buffer and $\mathrm{Q}_{2}$ the ethanol phase containing the lipids. $\mathrm{C}$ - Three dimensional view of the device, showing the wedges, associated to the moulding technique of microfabrication. The dimensions of the channels were measured by optical profilometry (see Fig 2).

The system includes a series of four tori, connected by straight channels. The device is microfabricated with plastic moulding technology (Ignite ${ }^{\circledR}$ from Precision NanoSystems Inc. ,Vancouver, BC, Canada). Figure 2 shows the cross-section of the outlet channel, located downstream of the four rings, as shown in the inset (see the white dashed line).

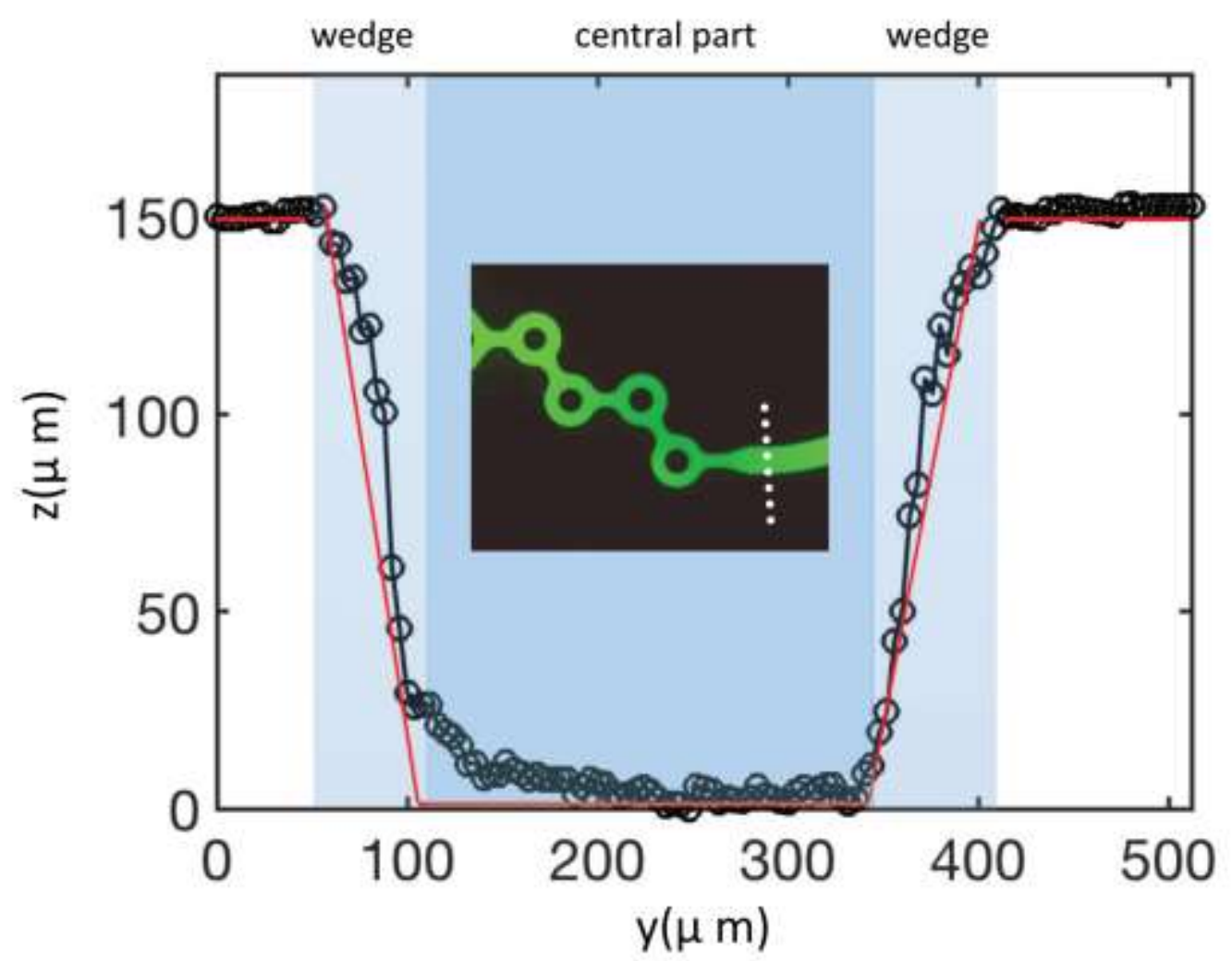

Figure 2: Cross-sectional profile of the outlet channel (see the dashed line in the insert), with $y$ the horizontal dimension (i.e. in the plane of the device) and $z$ the height, the function $z(y)$ thus defining the channel profile, with the channel bottom taken as a reference $(z=0)$. Two set of data are shown: intensity measurements 
(circles), assuming proportionality between the fluorescence intensity and $z(y)$, and Veeco optical profilometry measurements (red lines).

In Fig 2, the profile is obtained by filling the channels with a solution of alcohol and fluorescein, image them with a fluorescence microscope, collect the intensity field with a camera and analyze the images with a computer. The larger the channel height, the stronger the collected intensity. Working with low fluorescein concentrations $(0 / 1 \% \mathrm{w} / \mathrm{w})$ to avoid bleaching, we assume proportionality between the fluorescence intensity collected by the camera and the channel height. We checked that the profiles obtained in this manner are in full agreement with optical profilometry (see the red line of Fig 2, obtained with the optical profilometer Veeco). Because of the microfabrication technology used (injection moulding), the channels walls are not vertical, but slightly inclined. We obtain, from Fig 2, angles in the order of $80^{\circ}$, forming what we call "wedges", by opposition with the central part of the channel, shown in Fig 2, for which the height is constant. In this context, we define the channel width $w$, as the distance between the two inclined walls, at the mid-plane level. In our mixing analysis, we will neglect these wedges and concentrate on the central parts, which transports most of the material we want to produce (in the wedge, because of the confinement, the fluid, subjected to the same pressure gradient as in the central part, is essentially stagnant and we may assume that it does not contribute to the production process). The surface roughness, estimated from optical profilometry, is $3 \pm 1 \mu \mathrm{m}$. This is typical for injection technology, with no surface post-processing. The flow operating well below turbulence onset (see below), this roughness does not play any dynamical role.

The channels have the same measured depth, equal to $155 \mu \mathrm{m}$ (measured by optical profilometry), and different widths. In Fig 1, the tori are $150 \mu \mathrm{m}$ wide, the connecting channels $150 \mu \mathrm{m}$, and the entry and outlet channels $280 \mu \mathrm{m}$ wide (as shown in Fig 2). The device has two entries. The entry channels form a $\mathrm{Y}$, which is connected to the first torus.

The total flow-rate is defined by $Q=Q_{1}+Q_{2}$, where $Q_{1}$ and $Q_{2}$ are the flow-rates of the fluids injected at the two entries (See Fig 1). In our system, two types of experiments are conducted:

- Model fluid experiments: In this case, $\mathrm{Q}_{1}$ corresponds to DI water and $\mathrm{Q}_{2}$ to the fluorescein ethanol solution at $0.1 \%$ mass concentration.

- LNP experiments: In this case, $\mathrm{Q}_{1}$ represents the aqueous phase containing the nucleic acid in an acidic buffer and $\mathrm{Q}_{2}$ the ethanol phase with the four solubilized lipids. 
We also define the FRR, flow-rate ratio, by the relation $Q R=\frac{Q_{1}}{Q_{2}}$; Most of the work focuses on $\mathrm{FRR}=3$, i.e the water flow rate is three times the ethanol solution flow-rate. The total flow-rate $\mathrm{Q}$ varied from $0.2 \mathrm{ml} / \mathrm{min}$ to $20 \mathrm{ml} / \mathrm{min}$. We define a characteristic speed $\mathrm{U}$ as the total flow-rate $\mathrm{Q}$ divided by the inlet channel cross-section, i.e. juste before ring 1 (see Fig 1). The Reynolds and Dean numbers are defined by the following expressions:

$$
R e=\frac{U h}{v} \text { and } D e=R e \sqrt{\frac{h}{2 R}}
$$

In which $h$ is the channel height, $v$ the kinematic viscosity of water and $\mathrm{R}$ the curvature radius of the tori. With the flow-rates we span, the Reynolds numbers vary from 10 to 1100, while Dean numbers range between 4 and 400 . We are thus well below developed turbulence regimes, that might have requested a fine control of wall roughness. From a dimensional viewpoint, our system depends on these two numbers, along with aspect ratios, and a dimensionless number characterizing the diffusion process. The parameter space is large and in the paper we will plot the observables in function of the flow-rates (which, from a pratical viewpoint, are useful) without attempting to generalize the conclusions by using dimensionless numbers.

\subsection{Method of characterization of the mixing process}

For the fluid model experiments, the characterization of the mixing process is performed by using visualization techniques. Fluorescein is injected at one entry, the fluid injected at the other entry containing no dye. The system is observed with a fluorescence microscope, equipped by a source at $480 \mathrm{~nm}$, and a camera band-filtered at $520 \mathrm{~nm}$, i.e around the emission peak.

As classically done in the literature ${ }^{19}$, the study of the fluorescence intensity field provides information on mixing. Similarly, we define a mixing index $H$ by the formula:

$$
H=1-\sqrt{\frac{\int_{0}^{w}\left(I-I_{\text {mean }}\right)^{2} d y}{w I_{\text {mean }}^{2}}}
$$

in which $y$ is the coordinate transverse to the flow, $w$ the channel width, $I_{\text {mean }}$ the averaged intensity across the channel width. By definition, when $H$ is close to unity, mixing is high. In the opposite case ( $H$ small) mixing is low. 


\section{Results}

\subsection{Results on the flow patterns and the mixing characteristics for FRR=3 in the fluid model experiments}

We first consider low flow-rates, keeping the flow-rate ratio FRR equal to 3 . The choice of this value is motivated by the fact, as will be shown later, that it enables the formation of LNP with the appropriate sizes for DNA or RNA delivery (around 100nm), a narrow size distribution and an excellent encapsulation efficiency. A typical image of the fluorescence field at low flow-rates is shown in Fig 3A.
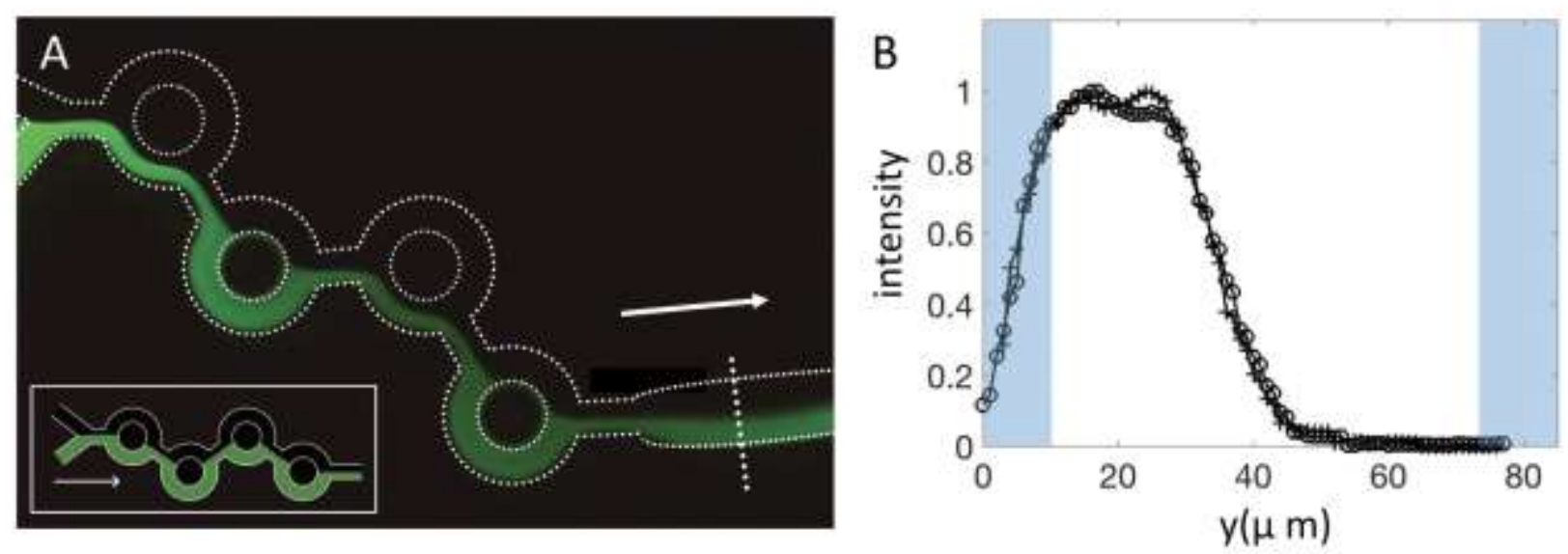

Figure 3: A - Fluorescence field of the micromixer, for a total flow-rates Q equal to $0.2 \mathrm{ml} / \mathrm{min}$. Insert: Theoretical tracer distribution, if the dye followed, without diffusion, the streamlines, for which we assume no recirculation. $\mathrm{B}$ - Intensity profiles in the outlet section of the system, for $\mathrm{Q}=0.2$ (open circle) and 0.3 (crosses) $\mathrm{ml} / \mathrm{min}$. Dashed line shows the center of the diffusive layer, and the grey areas the wedges, discussed in the Material section. The measurements are performed in the outlet channel, i.e downstream the fourth ring (see the dashed line). Grey zones are the channel wedges.

In Fig 3, the flow-rate $\mathrm{Q}_{2}$ of the ethanol solution is $0.05 \mathrm{ml} / \mathrm{min}$, while water is injected at a flow-rate $\mathrm{Q}_{1}$ equal to $0.15 \mathrm{ml} / \mathrm{min}$, so that the total flow-rate $\mathrm{Q}=\mathrm{Q}_{1}+\mathrm{Q}_{2}$ is $0.2 \mathrm{ml} / \mathrm{min}$, with, as said above, a flow-rate ratio FRR equal to 3. Fig. 3 indicates that, in the collecting entry channel, the two fluids travel side by side. The intensity profile shown in Fig 3B shows the existence of a diffuse layer. The measured thickness of the diffuse layer is on the order of $60 \mu \mathrm{m}$. It is larger, but of the same order of magnitude as the estimate $l \sim 6 \sqrt{D T}$ of diffuse layers, based on error function (with $\mathrm{D}$ is the fluorescein diffusion constant in ethanol $\left(210^{-10} \mathrm{~m}^{2} / \mathrm{s}\right)$ and $\mathrm{T}$ the travel time $(30 \mathrm{~ms}$ for $0.2 \mathrm{ml} / \mathrm{min})$ ). 
The formula leads to a thickness $l$ on the order of $30 \mu \mathrm{m}$. We hypothesize that the factor of 2 is due to the action of weak recirculations, always present in the system, perhaps localized at the entry, or developing along the rings. However small they can be, they may significantly enhance the diffusive transport.

Fig $3 \mathrm{~A}$ thus shows that ethanol and water flow side-by-side. In such a regime, the following expressions hold ${ }^{19,20}$.

$$
w_{e}=\frac{w}{1+\alpha}, w_{w}=\frac{\alpha w}{1+\alpha} \text { with } \alpha=\frac{\mu_{w} Q_{1}}{\mu_{e} Q_{2}}
$$

in which $\mu_{\mathrm{e}}$ and $\mu_{\mathrm{w}}$ are, respectively, ethanol and water viscosities. The formula says that fluorescein ethanol mixture occupies $30 \%$ of the channel width. This compares well with Fig 2, in which the dashed line, which marks the center of the diffuse layer, is located at $35 \%$ of the channel width. The insert of Fig 3A shows the flow pattern we would expect if there was no swirling motion and if diffusion was neglected. One sees an excellent correspondance between this pattern and the observed image.

As the total flow-rate $\mathrm{Q}$ is increased in the range $0.7-4 \mathrm{ml} / \mathrm{min}$, still with $\mathrm{FRR}=3$, the time averaged concentration profiles adopt complicated shapes, that substantially vary from one flow-rate to the other, even though the differences between two successive values are as small as $10 \%$. There is thus a strong variability in this region. We call this domain 'transition domain' and the corresponding regimes 'transition regimes'. Fig. 4B shows an example of a concentration profile obtained in this regime, measured, again, in the outlet microchannel (see the arrow).
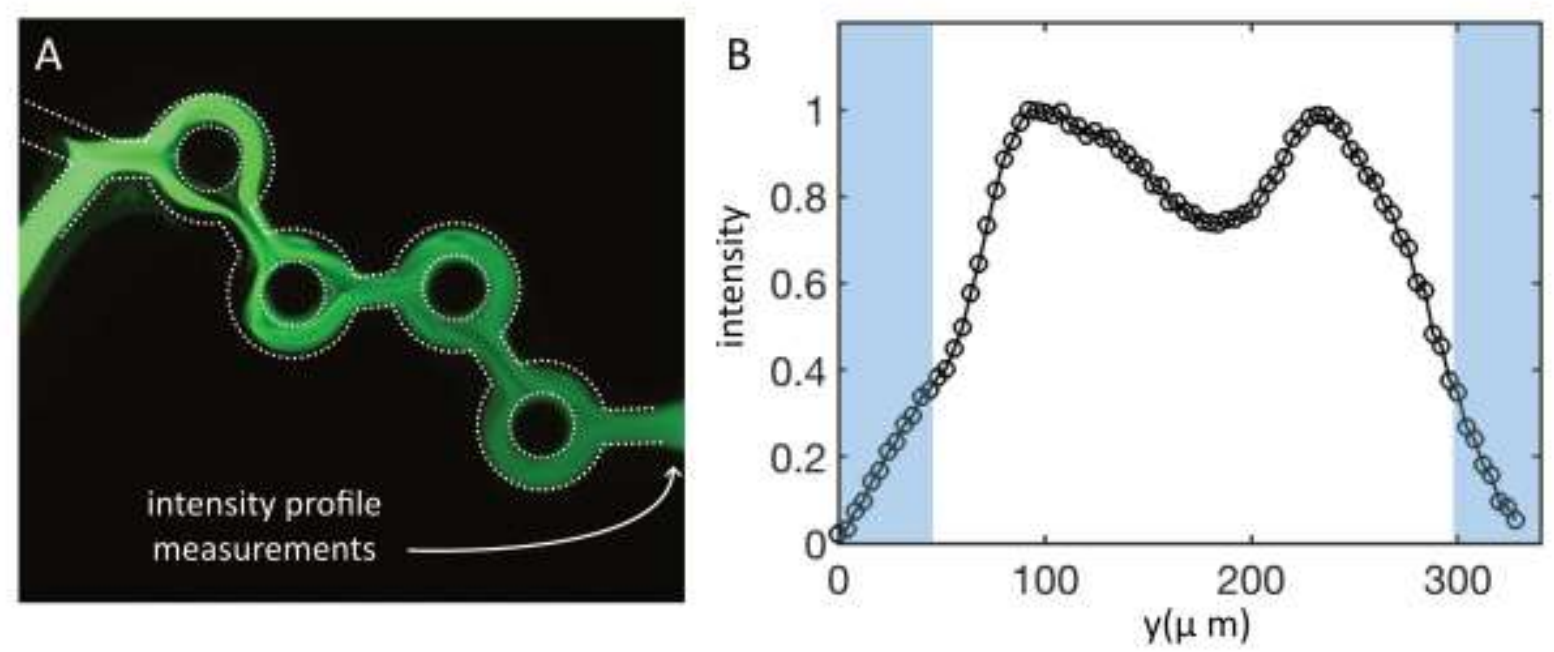

Figure 4: Fluorescence field of fluorescein transported, in the device, by ethanol, for $\mathrm{Q}_{1}=1.5 \mathrm{ml} / \mathrm{min}$ (water), $\mathrm{Q}_{2}=0.5 \mathrm{ml} / \mathrm{min}$ (ethanol): A - Instantaneous image of the fluorescence field. B - Time- 
averaged (over one minute) intensity profile, measured after the fourth ring (see the arrow), i.e. in the outlet channel. . Grey zones are the channel wedges.

In this case, the tracer invades the channel, but not totally: the concentration profile, which is averaged in time for one minute, shows a pronounced dip around the centerline. The mixing is therefore not complete, because some regions are well mixed, others less. One important observation is that, in the transition regime, fluorescein invades the channel before penetrating the first torus. This suggests that a mechanism of transport, conveyed by eddies developing at the junction where the two fluids meet, takes place. As indicated in Fig 3A, the dye concentration tends to homogeneize as we move downstream, but still, heterogeneities remain up to the outlet channel, as shown in Fig 3B.

An interesting feature that we observed in the $1-4 \mathrm{~mL} / \mathrm{min}$ range is the presence of time-dependent phenomena, shown in Fig 5.
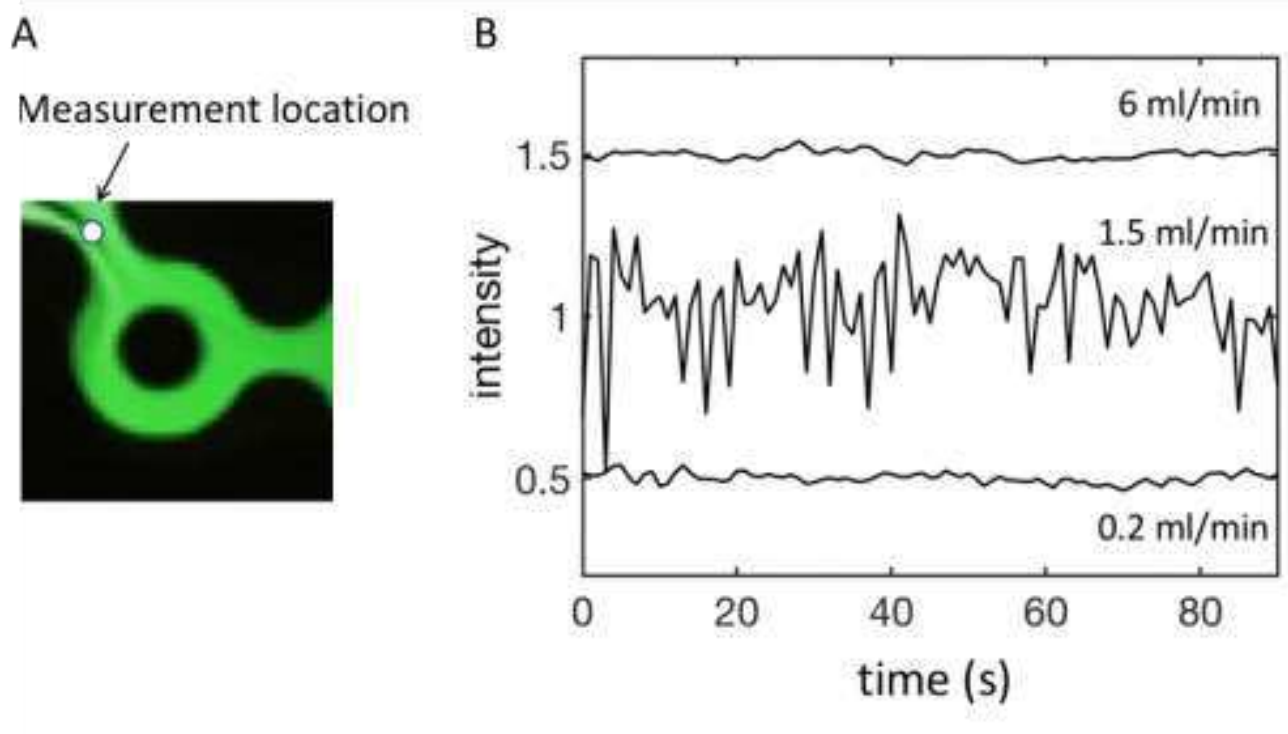

Figure 5: A - Instantaneous fluorescence image taken just before the Ring 2 for $Q=1.5 \mathrm{ml} / \mathrm{min}$, always keeping the FRR equal to 3. B - Instantaneous intensity measured at the point indicated by the arrow on the left figure. The positions of the sharp interfaces vary in time, giving rise to the oscillations in the local fluorescence intensity appearing on the intensity-time plot. The Reynolds numbers corresponding to the flow-rates shown on the plot are, from the lowest to the highest flowrates, 11,80 and 330 , respectively.

To obtain Fig 5, we measured the instantaneous intensity of fluorescence, at a fixed point, for different flow-rates. The position of the measurement point is indicated with a white dot on the left figure. Fig 5 (right) shows that at $0.2 \mathrm{ml} / \mathrm{min}$, there is no significant oscillation of the local intensity. At 1.5 $\mathrm{ml} / \mathrm{min}$, local oscillations, induced by fluorescein/water interface displacements, are visible. Their 
amplitude vanishes at larger flow-rates (see the curve at $6 \mathrm{ml} / \mathrm{min}$ ). These measurements convey evidence for the presence of time-dependent phenomena in the system. They develop in a narrow range of flow-rates, located, roughly, between 1 and $4 \mathrm{ml} / \mathrm{min}$. We hypothesize that, at larger flowrates, the flow is still unsteady, but the coupling between the hydrodynamic oscillations, which tend to striate the fluorescence field, as is currently observed in chaotic mixing ${ }^{19}$, and molecular diffusion, which efficiently smoothes it out, eventually produces a homogeneous concentration field. The limiting case of complete mixing is well illustratred in Fig 6A.
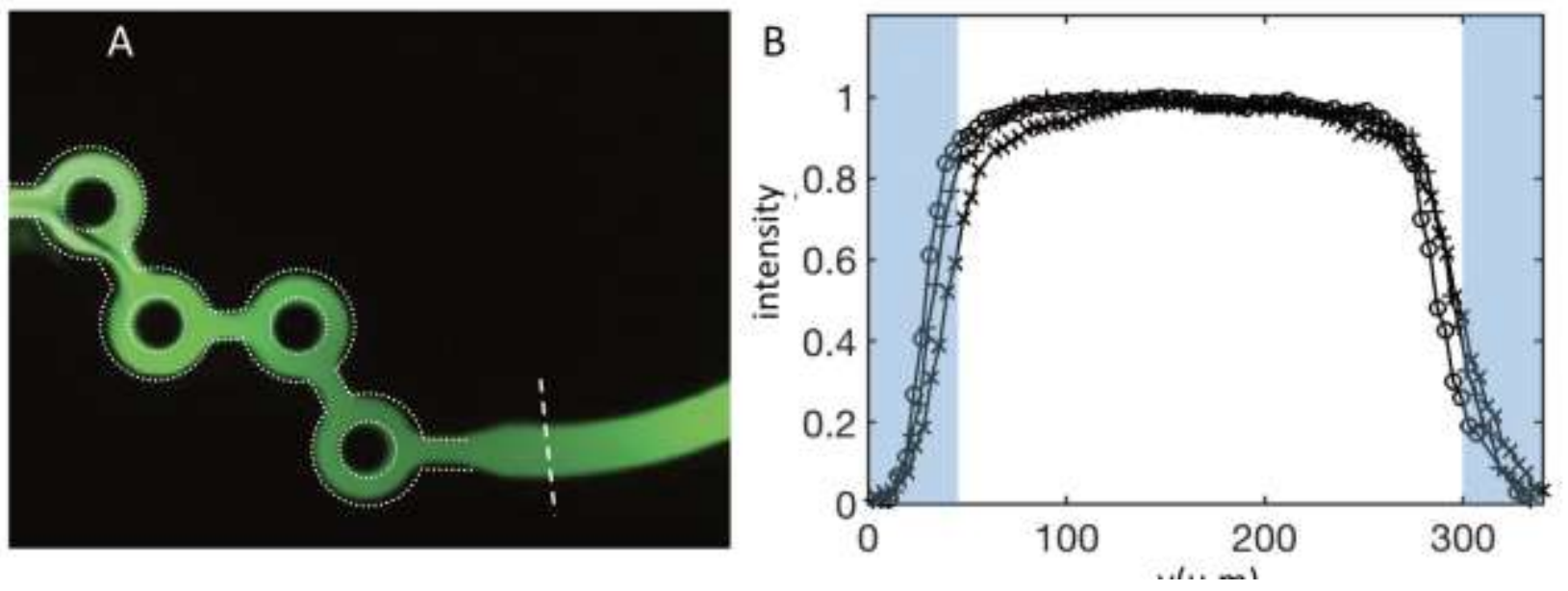

Figure 6: A - Fluorescence field developed by the micromixer, for $\mathrm{Q}=20 \mathrm{ml} / \mathrm{min}$; $\mathrm{B}$ - Three timeaveraged concentration profiles, obtained, respectively for Q equal to 6 (circles),14 (crosses) and 20 (plus) $\mathrm{ml} / \mathrm{min}$. Grey zones are the channel wedges.

The figure shows that the tracer, apart from a small region around the first ring, is homogeneously spread in the device. The corresponding concentration profiles are shown in Fig 6B, for three flowrates, 6, 14 and $20 \mathrm{ml} / \mathrm{min}$, still keeping $\mathrm{FRR}=3$. These profiles, again measured at the level of the collecting channel, close to the outlet (see the dashed line on Fig 6A), collapse onto each other.

Fig 7 represents the evolution of the homogeneity factor $\mathrm{H}$, defined precedently, measured in the outlet channel, for a range of flow-rates embracing the three regimes we singled out, keeping again the flow-rate ratio $\mathrm{FRR}=\mathrm{Q}_{1} / \mathrm{Q}_{2}$ equal to 3 . 


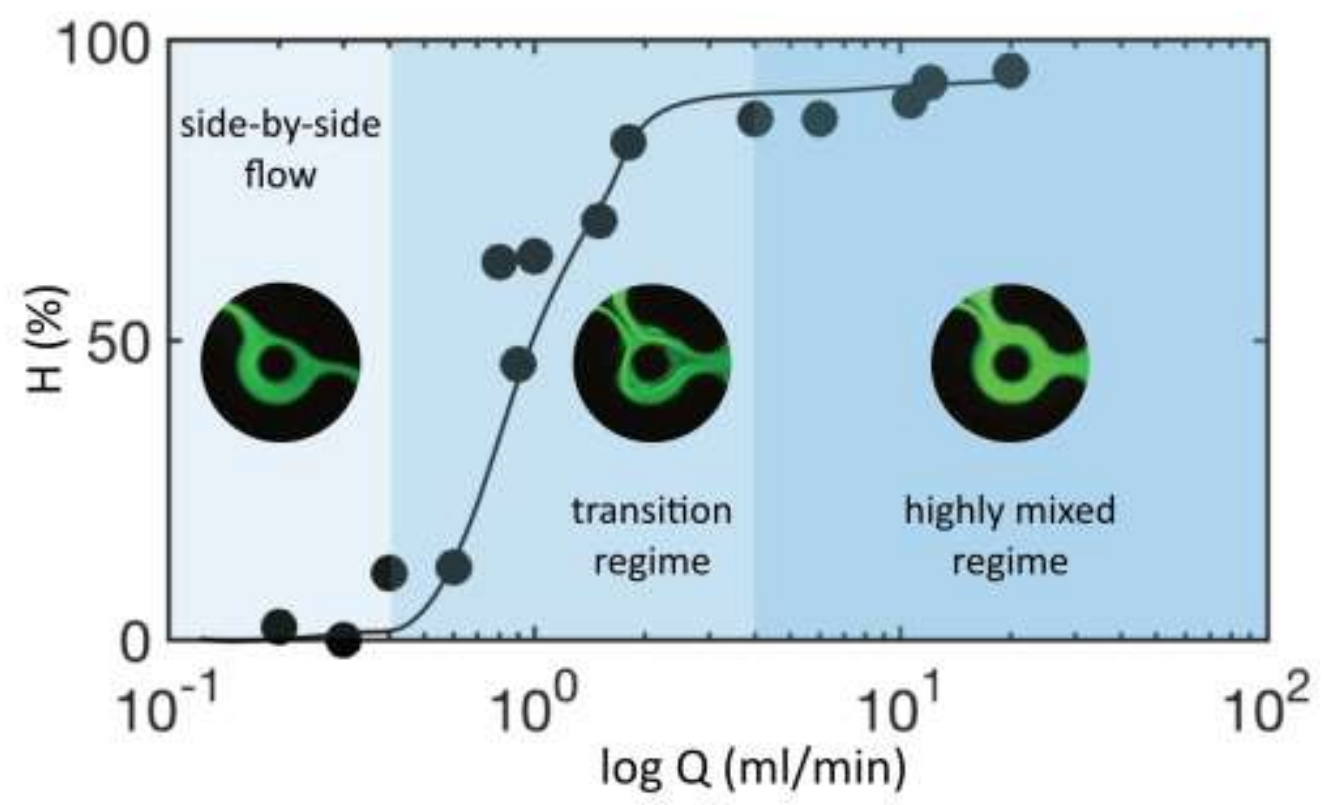

Figure 7: Evolution of the mixing index $\mathrm{H}$ as a function of the total flow-rate $\mathrm{Q}$, for a FRR equal to 3, with typical instantaneous images of fluorescein. Each point results from an average of one minute. The full line has been plotted to guide the eyes.

The data is plotted on a semi-logarithmic scale and the homogeneity factor $\mathrm{H}$ is obtained by excluding the wedges, considering, as said above, that they do not significantly contribute to the production process. The three regimes we identified from these measurements are the following (see Fig 8):

- $\quad \mathrm{Q}<0.4 \mathrm{ml} / \mathrm{min}$ Poor mixing, associated to side-by-side flows. The homogeneity factor $\mathrm{H}$ is small, a few percents or so. The corresponding upper Reynolds number of this regime is 22 .

- $\quad 0.4<\mathrm{Q}<4 \mathrm{ml} / \mathrm{min}$ : Transition regime, associated to moderate mixing, along with complex concentration profiles and time dependent phenomena. The homogeneity factors $\mathrm{H}$ lie between 20 and $80 \%$. In terms of Reynolds numbers, the domain ranges between 22 and 220 . The dispersion of $\mathrm{H}$, from one measurement to the other, even though they are averaged over long times, compared to the characteristic time of the oscillation, is typical of systems in which flow instabilities develop. This dispersion agrees with remarks made above concerning the variability of the concentration profiles with the flow conditions.

- $\mathrm{Q}>4 \mathrm{ml} / \mathrm{min}$, highly mixed regime with homogeneity factors above $80 \%$ and flat, reproducible, concentration profiles. The Reynolds number range lies between 220 and the maximum value reached in the experiment, i.e. 1100.

For discussing Fig. 7, it is interesting to split the system in two parts, as sketched in Fig 1B: 
- Section 1: The entry region, including the $\mathrm{V}$ junction and the channel of connection to the first ring.

- Section 2: The rest of the system, including the rings, the junctions between two successive rings and the outlet microchannel.

Section 1: Our observations can be compared to Minakov ${ }^{18}$, in which two miscible fluids are injected, at a T-junction, into a straight channel. The geometry is thus similar to ours, except that we have a Y-junction rather than a T-one. The results of this work were qualitatively confirmed experimentally $^{21}$. According to these references, above a certain threshold estimated to $\operatorname{Re} \approx 20$, symmetric, S-shaped, Dean type vortices develop in the junction. This threshold is close to ours (estimated to 22 ). Above this regime, an asymmetric steady pattern develops, up to $\operatorname{Re} \approx 150$. The development of oscillatory instabilities appears at 240, inducing a periodic displacement of a saddle point, which, according to Poincaré-Melnikov scenario, give rise to chaotic mixing. In effect, Minakov's work shows efficient mixing just after the onset of the time-dependant flows ${ }^{18}$. This range of Reynolds number is consistent with the regime we called 'transition regime', in which side-flow regime does not hold, mixing is moderate, heterogeneities are present, and oscillations develop. In our case, the transition regime extends from a Reynolds number equal to 22 to 220 , which is not as broad as Minakov's results, but consistent with it. We may thus suggest that our 'transition regime' embraces shaped eddies, assymetric eddies and oscillating eddies. Oscillating eddies are known to be extremely efficient from the point of view of mixing ${ }^{19}$. This may explain why, just above the onset of oscillations, we reach what we called 'highly mixed regimes'. So comparison with the work of Minakov provides clues to understand, on a semi-quantitative basis, the behavior of our micromixer.

Section 2: There is evidence that the four rings improve dye homogeneization. This is illustrated on Fig 5, which shows that the inhomogeneities at the entry of the device tend to be smoothed out as we move downstream. In the rings, two parts can be singled out: one is the ring itself (Part 1), the other (Part 2) the collecting channel, i.e. the region where the two fluids meet again. Part 1 presumably plays a minor role regarding mixing, because, as compared to the junctions (i.e Part 2 or the V entry), the curvature is smaller, and therefore the Dean vortices that may develop are weaker. On the other hand, Part 2 may be regarded as a Y-junction, playing a role similar to the entry, thus improving mixing. In fact, the experimental observations we made suggest that mixing events first start at the entry, i.e in Section 1 and repeat in the Parts 2 of the rings. So, we can suggest that Section 2 complements the work done by Section 1, regarding mixing, but does not initiate it. 


\subsection{Morphology of nanoparticles in the highly mixed regime with FRR $=3$}

We analyze here the morphology of the nanoparticles produced in the highly mixed regimes, at 4 $\mathrm{mL} / \mathrm{mn}$ and FRR=3. We used cryo-TEM, considering, for the sake of comparison, LNP without pDNA (“empty” LNP) and with nucleic acid (pDNA LNP). This is shown in Fig 8.

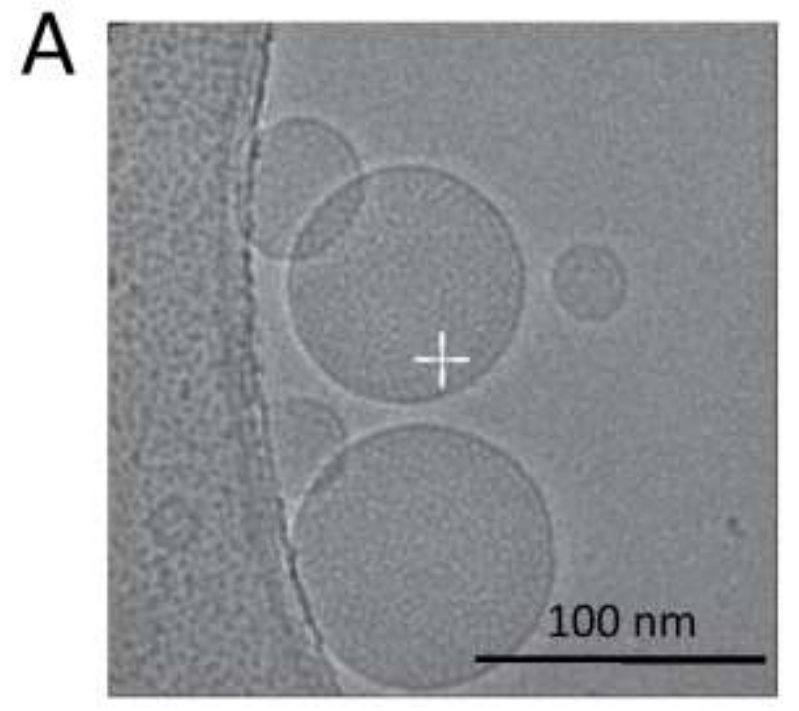

Empty LNP
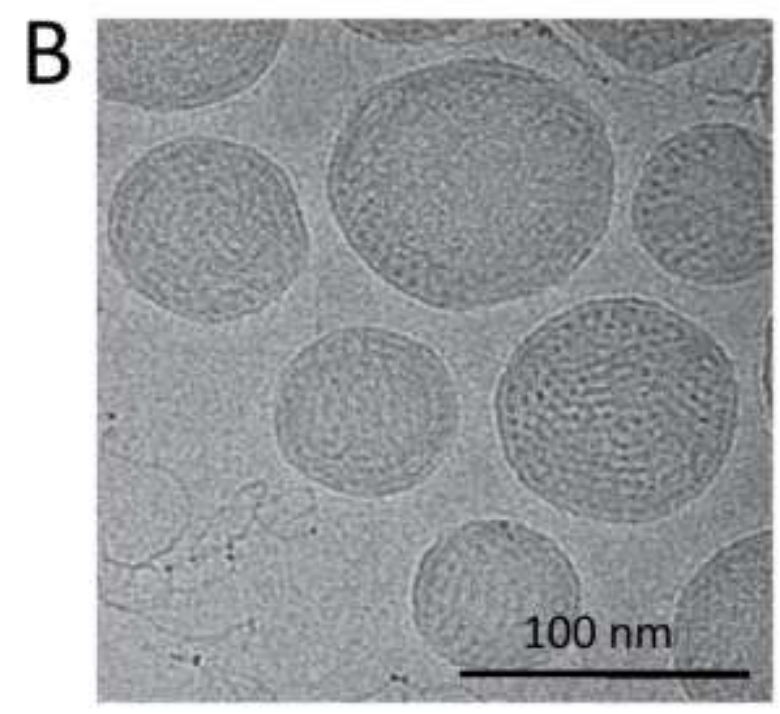

pDNA LNP

Figure 8: Cryo-TEM images of LNP composed of MC3, DOPC, PEG-Lipid and cholesterol with a molar composition of 50/10/1.5/38.5, respectively, and obtained at large flow-rates $(4 \mathrm{~mL} / \mathrm{min})$ and with FRR of 3. A - LNPs prepared in the absence of nucleic acid. B - pDNA-LNP systems prepared with p-DNA, showing more textured surfaces. Scale bar $=100 \mathrm{~nm}$.

The size (close to $100 \mathrm{~nm}$ ), observed by Cryo-TEM is consistent with DLS measurements. We find that empty LNP have smooth spherical shapes whereas pDNA LNPs show a corrugated interface (Figure 8B). It is probable that the action of encapsulated DNA strands on the lipid layer is at the origin of this phenomenon. In any case, we find that the electron dense core structure of Fig 8 agrees well with functional acid-based LNPs reported in the literature ${ }^{5,8,22,23}$. We may thus conclude that in highly mixed conditions, and with $\mathrm{FRR}=3$, the structures we found are 'optimal', in the sense that they are consistent with the functional LNPs imaged in the literature. 


\subsection{The correlation between mixing and LNP characteristics for a flow-rate ratio FRR equal to 3.}

The question we address now is the connection between the mixing characteristics, as analysed precedently, and the LNP properties, obtained with the same micromixer. We thus investigated the effect of the total flow-rate Q on the LNP size, size variability (PDI - Polydispersivity Index) and encapsulation efficiency (EE). This work represents the 'LNP experiments' we previously defined. The above characteristics are important for the usage of LNP: according to the literrature, ideally, LNP sizes must be around $100 \mathrm{~nm}$ or less to ensure optimal biodistribution and efficient drug delivery $^{24}$, PDI lower than 0.2 to certify the product homogeneity and encapsulation efficiency as high as possible to guarentee high yield of the process and the protection of the nucleic acid. In order to analyze the correlation of these parameters with the flow conditions, five flow-rates $\mathrm{Q}$, ranging from $0.4 \mathrm{ml} / \mathrm{min}$ to $20 \mathrm{ml} / \mathrm{min}$ were investigated. In these experiments, in the same manner as above, the ratio between aqueous and solvent streams was maintained equal to 3 and the lipid concentration $(1.44 \mathrm{mg} / \mathrm{l})$ and the chemical composition were kept constant. The data is shown in Fig 9.
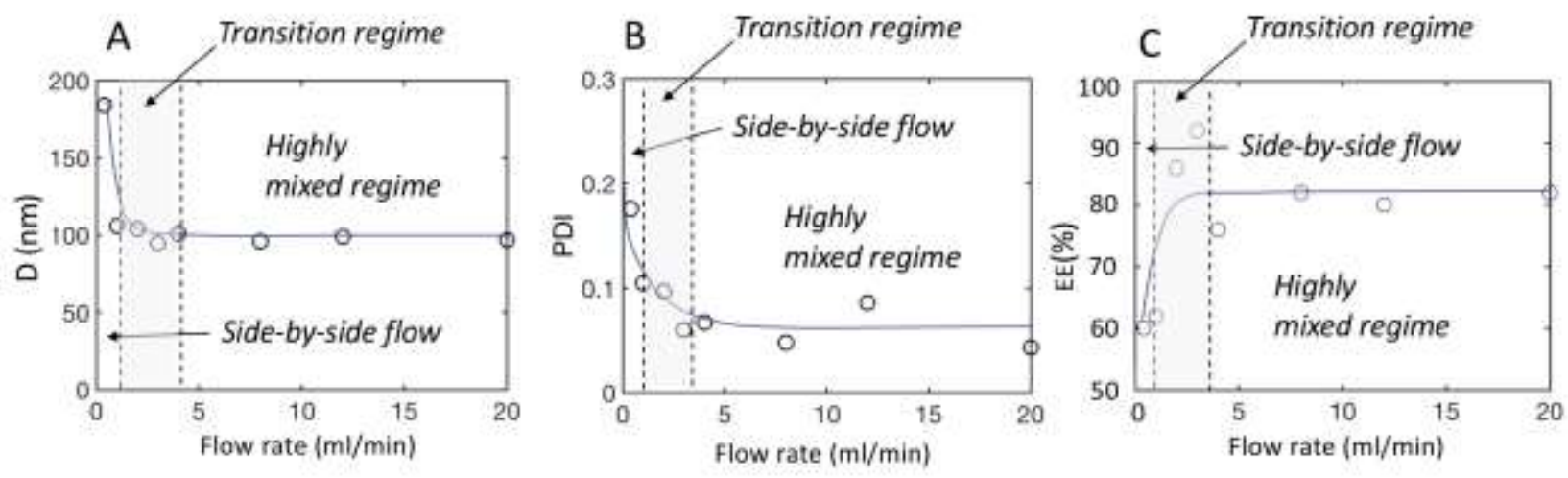

Figure 9: Evolution of various quantities with the flow-rate, between 0.4 to $20 \mathrm{~mL} / \mathrm{min}$ A- LNP Sizes; B- PDI (Poly Dispersvity Index of the LNP) . C- Encapsulation efficiency.

Figure 9A shows that larger particles were obtained at low Q $(<1 \mathrm{ml} / \mathrm{min})$. After a transition zone where the sizes seem to decrease, the particle sizes reach a plateau, located around $100 \mathrm{~nm}$ at larger flow-rates, i.e between 4 to $20 \mathrm{ml} / \mathrm{min}$. In the meantime, the nanoparticule size dispersivity (PDI) decreases as the flow rate is increased, levelling off, for $\mathrm{Q}>4 \mathrm{ml} / \mathrm{min}$ at $7 \%$ (see Fig 9B). In parallel, the EE is about $60 \%$ below $2 \mathrm{ml} / \mathrm{mn}$, while particles formed at higher Q, above $4 \mathrm{ml} / \mathrm{min}$, have an $\mathrm{EE}$ around $80 \%$ (see Fig 9C). These results correlate well with the mixing study we performed. We have plotted, on the same graph, the boundaries of the three zones we singled out in Fig 6, i.e the poorly 
mixed, transition and highly mixed regions. It is remarkable that the structure characteristics of the LNP correlate well with the mixing characteristics of the device. We may conclude, from a practical prospective, that when the mixing is not satisfactory (i.e with a homogeneity index $\mathrm{H}$ below $80 \%$ ), the properties of the structures that are created are not optimal, whereas they reach an optimum when mixing is high ( $\mathrm{H}$ larger than $80 \%$ ) and intensity profiles are homogeneous across the channel. We suggest that working above the transition zone, more specifically substantially above the conditions for which oscillatory flows develop, leads to obtain optimal LNPs, such as the one shown in Fig 8.

\subsection{Evolution of LNP characteristics with the flow-rate ratio (FRR)}

It is important, as was described in Roces, C. B. et al. ${ }^{5}$, to address the question of the role of the flowrate ratio FRR. Figure 10 shows results obtained on the LNP diameter D, the PDI, and the encapsulation efficiency EE, for a total flow-rate of $4 \mathrm{ml} / \mathrm{min}$, and different values of FRR, varying from 1 to 10.
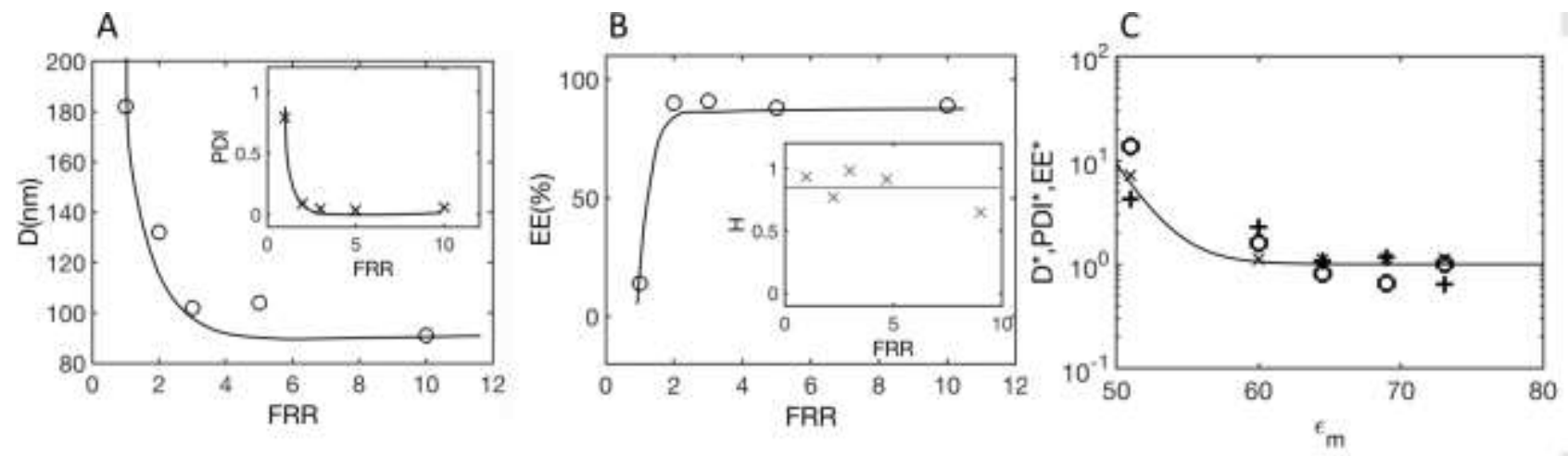

Figure 10 : Graphs obtained for a total flow-rate $Q=4 \mathrm{ml} / \mathrm{min}$. A - LNP diameter D as a function of FRR (i.e water over ethanol solutions flow-rate ratio); inset: PDI as a funcion of FRR; B- Encapsulation efficiency $\mathrm{EE}$ as a function of FRR. Inset: Homogeneity factor $\mathrm{H}$ as a functio of FRR ; C: Normalized quantities plotted as a function of $\varepsilon_{m}=\frac{F R R \varepsilon_{w}+\varepsilon_{e}}{F R+1} ; D^{*}=1+4 \frac{D-D_{\infty}}{D_{\infty}}(+) ; P D I^{*}=P D I / P D I_{\infty}(\mathrm{o})$ and $E E^{*}=E_{\infty} / E E(\mathrm{x})$.

For FRR smaller than 2, the LNP characteristics are not 'optimal'(in the sense defined above) : diameters above $100 \mathrm{~nm}$ (Fig 10A), low encapsulation efficiency (inset of Fig 10A) and large PDI (Fig 10B) are observed. At larger FRR, we recover the optimal characteristics, i.e diameters close to $100 \mathrm{~nm}$, large EE and small PDI (on the order of 6\%). It is important to note, as shown by the insert of Fig 10B, that in all cases, i.e. at all FRR, mixing is high: the homogeneity factors $\mathrm{H}$ are, in the average, around $80 \%$, We may infer that the origin of the structural pathologies of the LNP, for FRR $<2$, are not due to insufficient mixing. 
Figure $10 \mathrm{C}$ allows to propose an explanation. When ethanol (relative dielectric constant $\varepsilon_{\mathrm{e}}=25$ ) and water (relative dielectric constant $\varepsilon_{\mathrm{w}}=78$ ) are mixed, the effective (relative) dielectric constant of the mixture is equal to $\varepsilon_{m}=\frac{F R R \varepsilon_{w}+\varepsilon_{e}}{F R+1}$. Fig $10 \mathrm{C}$ shows the evolution of the diameter D, PDI and encapsulation efficiency EE, in normalized forms, as a function of $\varepsilon_{m}$. Specifically, we represented the following quantities: $D^{*}=1+4 \frac{D-D_{\infty}}{D_{\infty}}, P D I^{*}=P D I / P D I_{\infty}$, and $E E^{*}=E_{\infty} / E E$, where ' $\infty$ ' is the value obtained at FRR $>4 \mathrm{~mL} / \mathrm{min}$. At high FRR, $\varepsilon_{m}$ is close to water, and LNPs have optimal properties, while at small FRR, $\varepsilon_{m}$ is substantially smaller, the energy landscape 'seen' by the LNP constituents is changed, and we may hypothesize that this affects in a detrimental manner the self assembling process and thereby LNP morphologies This type of situation occurs when important quantities of ethanol is used in the formulation ${ }^{22,25-27}$. From Fig. 9C, we estimate that the cross-over between the non optimal and optimal cases is located around an effective relative dieledctric constant $\varepsilon_{m}$ close to 60 , which corresponds to a flow-rate ratio FRR on the order of 2 , still for $\mathrm{Q}=4 \mathrm{ml} / \mathrm{min}$. From this remark, we may suggest that, optimally, FRR must be large enough to maintain the polarity of the solution close to that of water, and in the meantime small enough to avoid reaching high dilutions, that would lead to a decrease of the yield of the process.

\subsection{Discussion}

The results presented above indicate that acceptable LNP quality (low size, low PDI and high EE) is obtained when two conditions are met: a homogeneous mixture of the two phases used for formulating the LNP, with a homogeneity factor larger than $80 \%$ and a phase distribution such that the mean relative dielectric constant of the mixture is larger than 60 . This is the range of conditions we may recommend for preparing functional LNPs. Our results suggest that, independently of the mixer geometry, if one of the two conditions is not satisfied, LNP sizes, size homogeneity and encapsulation efficiency substantially differ from optimal values.

It is interesting to ask why, at low flow-rates, anomalous LNPs are obtained i.e. possessing significantly larger sizes and smaller encapsulation efficiencies than the optimal structures. The fluorescence profile of Fig. 3 shows the presence of a diffuse layer, $60 \mu \mathrm{m}$ thick, that separates the two fluids. In the center of this layer, the water/ethanol ratio is roughly $1: 1$. We may infer that, in this region, optimal self-assembly conditions are not met. On the other hand, lipids and pDNA are more massive than water and ethanol, and therefore diffuse more slowly. There will be a laps of time for which lipids will feel this suboptimal environment, and thereby precipitate. This reasoning may 
provide an explanation for the anomalous sizes and the low performances observed in the poor mixing regimes.

Worth to be noted, at the highest flow-rates we imposed, LNPs keep their integrity, indicating that they do not breakup. This can be understood by noting that, despite the high speed (i.e. $\mathrm{cm} / \mathrm{s}$ ), on the scale of the LNP, i.e. $100 \mathrm{~nm}$, shear stresses are low. Should we increase the system size, there would be no difficulty to raise the flow-rate and thus the throughput, provided the shear stress 'seen' by the LNP remains at the same level as in our experiments, and the flow conditions remain below the turbulence onset.

Finally, by using the understanding we gained in the present work, we may attempt to define optimal conditions for producing LNPs, in the type of mixer we used. First, the flow-rate must be large enough to achieve high mixing. In our case, a minimum flow-rate of $4 \mathrm{ml} / \mathrm{min}$ can be proposed. Second, the device should be long enough for the mixing process to fully develop. Our work suggests that thirty times the transverse dimension is acceptable. Finally, FRR must be large enough to maintain the medium polarity close to water, without operating with high dilution factors, which would generate waste. In our system, we may propose a FRR on the order of 3.

\section{CONCLUSION}

The paper analyzes, in some depth, the mixing process developing along a device including a Y junction and four rings. In the LNP context, this has not been done yet. The device we analyze belongs to the category of inertial micromixers, i.e. micromixers taking advantage of the action of inertial (Dean) vortices, and, in the meantime, operating below the turbulence onset. These mixers are well adapted to high throughputs, a key condition for envisaging mass production. Such throughputs cannot be achieved, for instance, with the herringbone micromixer. In this paper, we showed that time dependent phenomena develop above a certain threshold, close to the numerical work of Minakov et $\mathrm{al}^{18}$. Our analysis of the mixing process, along with LNP measurements, indicate that the optimal conditions for producing functional structures require flow-rates substantially above the onset of time-dependent phenomena. The rationale is that, as established in the chaotic literature (see for instance Ref 19), oscillatory eddies give rise to efficient mixing. We suggest that the criterion we propose here (operate above the onset of oscillatory flow-regimes) is general. It should be useful for designing high throughput micromixers dedicated to LNP production. 


\section{Data availability}

The data sets analysed during the current study is available from the corresponding author on reasonable request

\section{Conflicts of interest}

No conflict of interest

\section{Support}

The word is supported by CNRS, PSL, IPGG platform, ESPCI and a grant by SANOFI.

\section{Acknowledgements}

The authors thank La Ville de Paris, SANOFI, ESPCI, CNRS, PSL and IPGG for their support. They thank the members of MMX group for discussions.

\section{References}

1. Hessel, V., Löwe, H. \& Schönfeld, F. Micromixers - a review on passive and active mixing principles. Chemical Engineering Science 60, 2479-2501 (2005).

2. Cai, G., Xue, L., Zhang, H. \& Lin, J. A Review on Micromixers. Micromachines 8, 274 (2017).

3. Zhang, J. et al. Fundamentals and applications of inertial microfluidics: a review. Lab Chip 16, 10-34 (2015).

4. Evers, M. J. W. et al. State-of-the-Art Design and Rapid-Mixing Production Techniques of Lipid Nanoparticles for Nucleic Acid Delivery. Small Methods 2, 1700375 (2018).

5. Roces, C. B. et al. Manufacturing Considerations for the Development of Lipid Nanoparticles Using Microfluidics. Pharmaceutics 12, E1095 (2020).

6. Cullis, P. R. \& Hope, M. J. Lipid Nanoparticle Systems for Enabling Gene Therapies. Mol Ther 25, 1467-1475 (2017).

7. Terada, T. et al. Characterization of Lipid Nanoparticles Containing Ionizable Cationic Lipids Using Design-of-Experiments Approach. Langmuir 37, 1120-1128 (2021).

8. Kulkarni, J. A. et al. On the Formation and Morphology of Lipid Nanoparticles Containing 
Ionizable Cationic Lipids and siRNA. ACS Nano 12, 4787-4795 (2018).

9. Noguchi, H. \& Gompper, G. Dynamics of vesicle self-assembly and dissolution. J. Chem. Phys. 125, 164908 (2006).

10. Maeki, M. et al. Understanding the formation mechanism of lipid nanoparticles in microfluidic devices with chaotic micromixers. PLoS One 12, e0187962 (2017).

11. Shepherd, S. J. et al. Scalable mRNA and siRNA Lipid Nanoparticle Production Using a Parallelized Microfluidic Device. Nano Lett. 21, 5671-5680 (2021).

12. Stroock, A. D. et al. Chaotic mixer for microchannels. Science 295, 647-651 (2002).

13. Ligrani, P. M. \& Niver, R. D. Flow visualization of Dean vortices in a curved channel with 40 to 1 aspect ratio. The Physics of Fluids 31, 3605-3617 (1988).

14. Gelfgat, A. Yu., Yarin, A. L. \& Bar-Yoseph, P. Z. Dean vortices-induced enhancement of mass transfer through an interface separating two immiscible liquids. Physics of Fluids 15, 330-347 (2003).

15. Mouza, A. A., Patsa, C.-M. \& Schönfeld, F. Mixing performance of a chaotic micro-mixer. Chemical Engineering Research and Design 86, 1128-1134.

16. Howell, P. B., Mott, D. R., Golden, J. P. \& Ligler, F. S. Design and evaluation of a Dean vortex-based micromixer. Lab Chip 4, 663-669 (2004).

17. Nivedita, N., Ligrani, P. \& Papautsky, I. Dean Flow Dynamics in Low-Aspect Ratio Spiral Microchannels. Sci Rep 7, 44072 (2017).

18. Minakov, A., Rudyak, V., Dekterev, A. \& Gavrilov, A. Investigation of slip boundary conditions in the T-shaped microchannel. International Journal of Heat and Fluid Flow Complete, 161-169 (2013).

19. Conlisk, A. T. Introduction to Microfluidics. By Patrick Tabeling. Oxford University Press,. Journal of Fluid Mechanics 570, 503-505 (2007).

20. Bruus, H. Theoretical Microfluidics. (Oxford University Press, 2007).

21. Hoffmann, M., Schlüter, M. \& Räbiger, N. Experimental investigation of liquid-liquid mixing in T-shaped micro-mixers using $\mu$-LIF and $\mu$-PIV. Chemical Engineering Science 61, 29682976 (2006).

22. Kulkarni, J. A. et al. Design of lipid nanoparticles for in vitro and in vivo delivery of plasmid DNA. Nanomedicine: Nanotechnology, Biology and Medicine 13, 1377-1387 (2017).

23. Patel, S. et al. Naturally-occurring cholesterol analogues in lipid nanoparticles induce polymorphic shape and enhance intracellular delivery of mRNA. Nat Commun 11, 983 (2020).

24. Samaridou, E., Heyes, J. \& Lutwyche, P. Lipid nanoparticles for nucleic acid delivery: Current perspectives. Adv Drug Deliv Rev 154-155, 37-63 (2020). 
25. Eygeris, Y., Patel, S., Jozic, A. \& Sahay, G. Deconvoluting Lipid Nanoparticle Structure for Messenger RNA Delivery. Nano Lett. 20, 4543-4549 (2020).

26. Kulkarni, J. A. et al. On the Formation and Morphology of Lipid Nanoparticles Containing Ionizable Cationic Lipids and siRNA. ACS Nano (2018) doi:10.1021/acsnano.8b01516.

27. Roces, C. B. et al. Manufacturing Considerations for the Development of Lipid Nanoparticles Using Microfluidics. Pharmaceutics 12, 1095 (2020). 